\section{Photocarcinogenicity of selected topically applied dermatological drugs: calcineurin inhibitors, corticosteroids, and vitamin D analogs}

Catharina M. Lerche, Hans Christian Wulf

Department of Dermatology, Copenhagen University Hospital, Bispebjerg,

Copenhagen, Denmark

\section{Abstract}

Topical therapies constitute the mainstay of dermatological treatments for skin disorders, such as atopic dermatitis, contact dermatitis, psoriasis, or acne. Since some of these diseases are often chronic, treatment duration may last for years and may even last the patient's entire lifetime. Obviously, such longterm therapy may raise safety concerns, which also include the potential photocarcinogenic effect. Most patients are exposed to ultraviolet radiation (UVR) during leisure, work, vacations, or in tanning beds. Additionally, the patients may receive UVR via UVB phototherapy or psoralens plus UVA radiation (PUVA). The use of immunosuppressant's, such as corticosteroids and calcineurin inhibitors, has markedly increased. Patients with skin diseases have benefited from both systemic and topical treatment of both new and established drugs. The issue of a black box warning by the US Food and Drug Administration has increased concerns about photocarcinogenesis, which raises the question: "Are these drugs safe?"

This review focuses on the mechanism of action and photocarcinogenic potential of commonly used topical treatments, such as corticosteroids, calcineurin inhibitors, and vitamin D analogs.

\section{Introduction}

The skin is the largest organ system of the human body providing a barrier to harmful environmental effects including infectious agents, ultraviolet radiation (UVR) and exogenous chemicals. UVR is the most important environmental factor in the pathogenesis of skin cancer in humans. ${ }^{1}$ Importantly, the effect of UVR may be enhanced by pharmaceuticals. ${ }^{2}$ Agents that enhance photocarcinogenesis can be defined as chemicals or treatments that shorten the time of onset to skin cancer devel- opment after a specific UVR dose. ${ }^{3}$ Skin cancers fall into three categories: basal cell carcinoma (BCC), squamous cell carcinoma (SCC) (non-melanoma skin cancer), and malignant melanoma. ${ }^{1,4}$ Solar UVR is a carcinogen in humans and is involved in the development of all three types of skin cancer.

This review focuses on commonly used topical treatments, such as corticosteroids, calcineurin inhibitors, and vitamin D analogs. The mechanism of action of these drugs goes through different pathways but they all interfere with the transcriptional activation of various cytokine genes that modulate immune response and inflammation.

\section{Topical calcineurin inhibitors}

The topical calcineurin inhibitors, tacrolimus and pimecrolimus, were approved for treatment of atopic dermatitis in 2000 and 2001 , and the long-term safety of these products has been widely debated. ${ }^{5-14}$ The topical calcineurin inhibitors tacrolimus, (FK506) and pimecrolimus, (SDZ981) belong to the ascomycin class of macrolactam immunosuppressive drugs and their mechanism of action has been reviewed. ${ }^{12,15-21}$ They function by inhibiting T-cell activation through the calcineurin pathway, thereby inhibiting the release of numerous inflammatory cytokines and preventing the cascade of immune and inflammatory signals. The chemical structures of pimecrolimus and tacrolimus are very similar and their overall mechanism of action is the same. ${ }^{22}$

The mechanisms of action of pimecrolimus and tacrolimus are very similar to that of the well known immunosuppressive drug, cyclosporine A (CsA).$^{15,20} \mathrm{CsA}$ and tacrolimus are used systemically for the treatment of organ transplant recipients. ${ }^{23}$ Systemic CsA is also used in dermatology for a range of autoimmune diseases, including psoriasis and atopic dermatitis. There are many adverse events associated with immunosuppressive treatment and there is a large body of evidence showing that skin cancer is the most common malignant condition in transplant recipients. ${ }^{23}$ Basal cell carcinoma (BCC) and squamous cell carcinoma (SCC) are the most common cancers after organ transplantation, affecting more than half of all Caucasian patients in the long term. ${ }^{23}$ UVR appears to be the highest risk factor since the highest incidence of skin cancer is in countries with high sun exposure, e.g. Australia, and the tumors mainly develop in sun-exposed areas. ${ }^{23}$ Furthermore, it is known that CsA inhibits DNA repair in human keratinocytes, fibroblasts and lymphoblasts. ${ }^{24,25}$ In addition, CsA promotes the spread of cancer in mice without an immune system, probably by increasing the production of transforming growth factor $\beta$, interleukin-6, and vascular endothelial growth factor that enhance angio-
Correspondence: Catharina M. Lerche, Copenhagen University Hospital, Bispebjerg, Department of Dermatology, D92,

Bispebjerg Bakke 23,

DK-2400 Copenhagen NV, Denmark.

E-mail: cler0001@bbh.regionh.dk

Key words: pimecrolimus, tacrolimus, calcineurin inhibitor, corticosteroid, vitamin D analog.

Contributions: both authors contributed to conception and design, acquisition of data, analysis and interpretation of data, and gave final approval of the version to be published. HCW revised the article critically for important intellectual content.

Conflicts of interest: the authors have no conflict of interest to disclose.

Received for publication: 31 August 2010. Accepted for publication: 2 September 2010.

This work is licensed under a Creative Commons Attribution 3.0 License (by-nc 3.0).

(C) Copyright C.M. Lerche and H.C. Wulf, 2010

Licensee PAGEPress, Italy

Dermatology Reports 2010; 2:e13

doi:10.4081/dr.2010.e13

genesis, tumor growth, and metastasis. ${ }^{23}$

Documented adverse photocarcinogenic events for systemic treatment raise concerns as to the safety of topical treatment. ${ }^{12}$ As a result, the US Food and Drug Administration (FDA) issued a black box warning in January 2006. The cause for this warning originated from case reports from patients, animal studies, lack of post-marketing studies, and the known mechanism of action of the drugs. ${ }^{10,17}$

The case reports from patients reported to the FDA until 2004 showed a total of 28 malignancies in topical calcineurin users, 10 of which were in pimecrolimus users and 19 in tacrolimus users (one common case). ${ }^{13,17}$ These malignancies occurred in 21 adults and in 7 children. $^{13}$ To give a sense of the use of topical calcineurin inhibitors, 3.2 million prescriptions were issued for topical tacrolimus from January 2001 to August 2004, and 7.7 million for topical pimecrolimus from January 2002 to August 2004. ${ }^{17}$

The animal experiments which led to the warning consisted of a 52-week photocarcinogenesis study in mice that showed a decrease in the median time to onset of skin tumor formation in pimecrolimus- and tacrolimus-treated mice when compared with the control mice, indicating an increase in photocarcinogenesis. However, there was no difference or protective effect in the median time to onset of skin tumor formation for the pimecrolimus $1 \%$, tacrolimus $0.03 \%$, and $0.1 \%$ formulations compared to the vehicle, indicating that the 
increased tumor formation observed was caused by the vehicle alone. ${ }^{6,7}$ Vehicle effects on photocarcinogenesis have been reported before. ${ }^{27,28}$ It is possible that a higher content of tacrolimus than that found in the commercial ointment might facilitate photocarcinogenesis. Indeed, a decrease in the median time to onset of skin tumor formation was shown for both the tacrolimus $0.3 \%$ and $1 \%$ formulations when compared with vehicle and UV controls. ${ }^{6}$ The commercial ointment contains maximally $0.1 \%$ tacrolimus. We have conducted a smilar study showing that pimecrolimus and tacrolimus did not decrease the time to tumor development. ${ }^{29,30}$ It showed that application of tacrolimus or pimecrolimus 3-4 hours before 2 , 4 , or 6 standard erythema dose (SED) of irradiation resulted in an unchanged or increased number of days to tumor onset. . $^{29,30}$

Also data on chemical carcinogenesis and in vitro tests are conflicting. ${ }^{31,32}$ Niwa et al. report that topical treatment with $0.1 \%$ tacrolimus ointment stimulates the development of papillomas and carcinomas in the skin in the DMBA/TPA two-step carcinogenesis mouse model..$^{31}$ On the other hand, Jiang et al. report an opposite effect of tacrolimus in the same test in CD-1 mice. ${ }^{32}$ An in vitro study has shown that tacrolimus inhibits repair of UV induced DNA damage and apoptosis ${ }^{25}$ which could be an explanation for the increased photocarcinogenesis. However, a short-term murine study has shown no increase in DNA damage formation after topical treatment with pimecrolimus or tacrolimus over a 10-day period followed by UVB irradiation, indicating a biologically pertinent protective effect with regard to UV-mediated DNA damage. ${ }^{33}$ It has also been shown that pimecrolimus does not enhance cyclobutane pyrimidine dimer formation in human skin one and 24 hours after UV exposure. ${ }^{34}$ These data support the conclusions of another study that showed no difference in the development of epidermal thymine dimers or increase in p53 levels at one and 24 hours after a 3-day pre-treatment of tacrolimus on human skin followed by UV exposure compared to vehicle or UV treatment alone..$^{35}$

Several epidemiological studies and recent reviews have shown no increased risk of skin cancer and lymphoma in patients treated with topical calcineurin inhibitors. ${ }^{36-40}$

Worldwide, more than 20 million prescriptions of topical calcineurin inhibitors have been issued. The safety aspects of the inhibitors have been under focus through assessment of pre-clinical, clinical, and postmarketing reports. However, pimecrolimus cream and tacrolimus ointment have been in clinical use for less than a decade, so investigation through long-term trials and phase IV post-marketing studies with large sample sizes and extended follow ups are still required. ${ }^{7}$

\section{Topical corticosteroids}

Skin photocarcinogenicity related to the use of topical corticosteroids has been a concern due to the immunosuppressive effect of this class of drugs. ${ }^{8}$ Use of systemic glucocorticosteroids has been associated with an increased risk of non-melanoma skin cancer in humans. ${ }^{41}$ Little information is available from studies on photocarcinogenesis mediated by topical corticosteroids. Instead, the safety record of topical corticosteroids has been assessed through many years of clinical data. $^{6,17}$ Topical corticosteroids have antiinflammatory, antipruritic, and vasoconstrictive properties and their mechanism of action has been reviewed..$^{18,19,42,43}$ At the cellular level, corticosteroids act directly by binding to a glucocorticoid receptor to form a steroid-receptor complex and this complex translocates into the nucleus. ${ }^{42}$ Inside the nucleus this complex binds to the glucocorticoid-responsive element and thereby decreases the transcription and protein synthesis of various cytokines. ${ }^{18}$ Indirectly, corticosteroids can act by, for example, the transcription factors activator protein

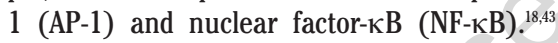
Corticosteroids have been shown to increase cellular levels of inhibitory nuclear factor- $\mathrm{K} \mathrm{B} \alpha$ (I $\kappa \mathrm{B} \alpha$ ) by stimulating expression of the I $\mathrm{B} \alpha$ gene. The I $\mathrm{I} \mathrm{B} \alpha$ protein binds to the transcription factor NF- $\kappa \mathrm{B}$ so the latter cannot translocate into the nucleus, which also results in a decreased transcription and protein synthesis of various cytokines. ${ }^{18}$ Corticosteroids decrease the synthesis of various proinflammatory cytokines such as IL-1, IL-2, IL-6, IFN- $\gamma$ and TNF- $\alpha \cdot \cdot^{18,19,43}$ In addition, corticosteroids stimulate the lymphocyte expression of genes for anti-inflammatory cytokines, including transforming growth factor- $\beta$ and IL-10. ${ }^{18}$ In the skin, corticosteroids also inhibit capillary dilation, dermal edema and reduce the number of Langerhans cells by inducing apoptosis. ${ }^{18,43,44}$ However, corticosteroids are also thought to act by second messengers (lipocortins) inducing antiproliferative effects. ${ }^{18}$ Topical steroids are well known to produce side effects related, at least in part, to their inhibition of epidermal proliferation and connective tissue synthesis. ${ }^{448}$ Four murine studies have demonstrated inhibition of photocarcinogenesis using different topical corticosteroids. ${ }^{49-52}$ One study showed that hydrocortisone protected against UVB radiation-induced tumor formation as measured by the time to tumor onset and number of tumors per mouse. ${ }^{51}$ Another study showed that diflorasone diacetate protected against SSR-induced tumor formation using almost identical measurement methods. ${ }^{49} \mathrm{~A}$ third study showed that triamcinolone acetonide protected against UVB-induced tumor formation, as measured by the number of surviving mice with tumors and the mean tumor diameters..$^{50}$ Our study showed that hydrocorti- sone butyrate and clobetasol propionate did not significantly decrease the time to tumor development. ${ }^{52}$ Hydrocortisone butyrate in combination with 2, 4 or 6 SED resulted in an unchanged or increased number of days to tumor onset. ${ }^{52}$ Clobetasol propionate in combination with 2 SED resulted in an unchanged number of days to the first tumor but an increased number of days to the second and third tumor. All other combinations of clobetasol propionate and 4 or 6 SED resulted in a significant photoprotective effect..$^{52}$ None of the published data on topical treatment of mice with corticosteroids has shown increased risk of photocarcinogenesis. Furthermore, topical corticosteroids are also known to inhibit twostage chemical carcinogenesis in mice. ${ }^{53}$ In agreement, no reports have been published concerning the use of topical corticosteroids and photocarcinogenesis in humans.

\section{Vitamin D3 analogs}

Chemically, the various forms of vitamin D are collectively called secosteroids, steroids in which one of the bonds in the steroid rings is broken. Calcipotriol, also called calcipotriene, is a synthetic vitamin D3 analog used in the treatment of psoriasis. ${ }^{54}$ The mechanism of action of calcipotriol has been reviewed. ${ }^{18,19,55}$ The mechanism acts through the inhibition of keratinocyte proliferation and improvement of keratinocyte differentiation. This is probably mediated via binding with vitamin D receptors located in the nucleus of epidermal keratinocytes. ${ }^{18}$ This receptor complex can regulate transcription by binding with elements in the promoter region of target genes and it can also interfere with other transcription factors such as those described previously, e.g. NF-AT and NF- $\kappa$ B. ${ }^{18,19}$ Vitamin D analogs have also been shown to decrease the synthesis of various proinflammatory cytokines, such as IL-2, IL-6, IL-8, IFN- $\gamma$ and GM-CSF, which are all a part of cutanous inflammation and proliferation of T lymphocytes and keratinocytes. ${ }^{18,19}$ Indirectly, vitamin D3 analogs can, like the corticosteroids, act by, for example, the transcription factor AP-1, which causes a negative regulation of IL-2 and GM-CSF. ${ }^{18}$

It has been demonstrated that calcitriol $\left(1,25(\mathrm{OH})_{2} \mathrm{D}_{3}\right)$, which is the active metabolite of calcipotriol, protects primary human keratinocytes and murine epidermis against the induction of cyclobutane pyrimidine dimers by UVR. ${ }^{56,57}$ Studies with calcitriol on murine chemical carcinogenesis have been inconclusive. One study showed an augmentation of carcinogenesis while another study showed a protective effect. ${ }^{58,59}$ The Summary of Product Characteristics refers to a study which indicated that calcipotriol can accelerate photocarcinogenesis in male albino mice. ${ }^{60}$ We showed that calcipotriol in combination with 2 SED decreased the time to the first tumor signifi- 
cantly and suggests that the photocarcinogenic effect was increased..$^{52}$ The other combinations of calcipotriol and 4 or 6 SED resulted in an unchanged or increased number of days to tumor onset..$^{52}$ To our knowledge, no reports have been published concerning the use of topical vitamin D analogs and photocarcinogenesis in humans.

\section{Conclusions}

Topical calcineurin inhibitors show conflicting photocarcinogenicity data from animal and in vitro studies. Nevertheless, more than 20 million prescriptions for pimecrolimus cream and tacrolimus ointment are issued worldwide, and several epidemiological studies and recent reviews have shown no increased risk of skin cancer and lymphoma in patients treated with topical calcineurin inhibitors. However, pimecrolimus cream and tacrolimus ointment have been available for less than ten years, so longterm studies and phase IV post-marketing studies with large sample sizes and extended follow up is still needed.

Even though topical steroids have been on the market for a very long time, only few photocarcinogenesis studies have been conducted. All published studies indicate that topical steroids do not increase the photocarcinogenic potential of UVR.

Only one study has been published regarding photocarcinogenesis and vitamin D analogs. The study indicates that calcipotriol does not increase the photocarcinogenic potential of UVR.

Based on the current data, the cancer risk associated with the use of topical calcineurin inhibitors, topical corticosteriods and vitamin D analogs on sun-exposed areas appears to be minimal or non-existent.

\section{References}

1 Rass K, Reichrath J. UV damage and DNA repair in malignant melanoma and nonmelanoma skin cancer. Adv Exp Med Biol 2008;624:162-78.

2 Epstein JH. Chemicals and photocarcinogenesis. Australas J Dermatol 1977;18:5761.

3 Forbes PD, Beer JZ, Black HS, et al. Standardized protocols for photocarcinogenesis safety testing. Front Biosci 2003; 8:d848-54.

4. Young AR. Cumulative effects of ultraviolet radiation on the skin: cancer and photoaging. Semin Dermatol 1990;9:25-31.

5. Wooltorton E. Eczema drugs tacrolimus (Protopic) and pimecrolimus (Elidel): can- cer concerns. CMAJ 2005;172:1179-80.

6. Ring J, Barker J, Behrendt H, et al. Review of the potential photo-cocarcinogenicity of topical calcineurin inhibitors: position statement of the European Dermatology Forum. J Eur Acad Dermatol Venereol 2005;19:663-71.

7. Ring J, Möhrenschlager M, Henkel V. The US FDA 'black box' warning for topical calcineurin inhibitors: an ongoing controversy. Drug Saf 2008;31:185-98.

8. Callen J, Chamlin S, Eichenfield LF, et al. A systematic review of the safety of topical therapies for atopic dermatitis. Br J Dermatol 2007;156:203-21.

9. Ashcroft DM, Dimmock P, Garside R, et al. Efficacy and tolerability of topical pimecrolimus and tacrolimus in the treatment of atopic dermatitis: meta-analysis of randomised controlled trials. BMJ 2005;330:516.

10. Berger TG, Duvic M, Van Voorhees AS, et al. The use of topical calcineurin inhibitors in dermatology: safety concerns. Report of the American Academy of Dermatology Association Task Force. J Am Acad Dermatol 2006;54:818-23.

11. Fonacier L, Spergel J, Charlesworth EN, et al. Report of the topical calcineurin inhibitor task force of the american college of allergy, asthma and immunology and the american academy of allergy, asthma and immunology. J Allergy Clin Immunol 2005;115:1249-53.

12. Weischer M, Röcken M, Berneburg M. Calcineurin inhibitors and rapamycin: cancer protection or promotion? Exp Dermatol 2007;16:385-93.

13. Ormerod AD. Topical tacrolimus and pimecrolimus and the risk of cancer: how much cause for concern? Br J Dermatol 2005; 153:701-5.

14. Stern RS. Topical calcineurin inhibitors labeling: putting the "box" in perspective. Arch Dermatol 2006;142:1233-5.[PubMed]

15. Stepkowski SM. Molecular targets for existing and novel immunosuppressive drugs. Expert Rev Mol Med 2000;2:1-23.

16. Grassberger M, Steinhoff M, Schneider D, et al. Pimecrolimus -- an anti-inflammatory drug targeting the skin. Exp Dermatol 2004;13:721-30.

17. Patel TS, Greer SC, Skinner RB Jr. Cancer concerns with topical immunomodulators in atopic dermatitis: overview of data and recommendations to clinicians. Am J Clin Dermatol 2007;8:189-94.

18. Norris DA. Mechanisms of action of topical therapies and the rationale for combination therapy. J Am Acad Dermatol 2005;53: S17-S25.

19. Bhatia N. Topical immunomodulation: modes of action with clinical correlation. Dermatol Clin 2007;25:147-55, vi.
20. Dé Tran QH, Guay E, Chartier S, Tousignant J. Tacrolimus in dermatology. J Cutan Med Surg 2001;5:329-35.

21. Marsland AM, Griffiths CE. The macrolide immunosuppressants in dermatology: mechanisms of action. Eur $\mathrm{J}$ Dermatol 2002;12:618-22.

22. Billich A, Aschauer H, Aszódi A, Stuetz A. Percutaneous absorption of drugs used in atopic eczema: pimecrolimus permeates less through skin than corticosteroids and tacrolimus. Int J Pharm 2004;269:29-35.

23. Euvrard S, Kanitakis J, Claudy A. Skin cancers after organ transplantation. N Engl J Med 2003;348:1681-91.

24. Kuschal C, Thoms KM, Mori T, et al. Cyclosporin A, but not everolimus, inhibits DNA repair in human fibroblasts and lymphoblasts. Int $\mathrm{J}$ Clin Pharmacol Ther 2009;47:38-40.

25. Yarosh DB, Pena AV, Nay SL, et al. Calcineurin inhibitors decrease DNA repair and apoptosis in human keratinocytes following ultraviolet B irradiation. J Invest Dermatol 2005;125:1020-5.

26. Hojo M, Morimoto T, Maluccio M, et al. Cyclosporine induces cancer progression by a cell-autonomous mechanism. Nature 1999;397:530-4.

27. Forbes PD. Relevance of animal models of photocarcinogenesis to humans. Photochem Photobiol 1996;63:357-62.

28. Sambuco CP, Forbes PD, Davies RE, et al. Photocarcinogenesis: measuring the reproducibility of a biologic response to ultraviolet radiation exposure in mice. Front Biosci 2003;8:a26-33.

29. Lerche CM, Philipsen PA, Poulsen T, Wulf HC. Topical tacrolimus in combination with simulated solar radiation does not enhance photocarcinogenesis in hairless mice. Exp Dermatol 2008;17:57-62.

30. Lerche CM, Philipsen PA, Poulsen T, Wulf HC. Topical pimecrolimus and tacrolimus do not accelerate photocarcinogenesis in hairless mice after UVA or simulated solar radiation. Exp Dermatol 2009;18:246-51.

31. Niwa Y, Terashima T, Sumi H. Topical application of the immunosuppressant tacrolimus accelerates carcinogenesis in mouse skin. Br J Dermatol 2003;149:960-7.

32. Jiang H, Yamamoto S, Nishikawa K, Kato R. Anti-tumor-promoting action of FK506, a potent immunosuppressive agent. Carcinogenesis 1993;14:67-71.

33. Tran C, Lübbe J, Sorg 0, et al. Topical calcineurin inhibitors decrease the production of UVB-induced thymine dimers from hairless mouse epidermis. Dermatology 2005;211:341-7.

34. Doelker L, Tran C, Gkomouzas A, et al. Production and clearance of cyclobutane dipyrimidine dimers in UV-irradiated skin pretreated with $1 \%$ pimecrolimus or $0.1 \%$ 
triamcinolone acetonide creams in normal and atopic patients. Exp Dermatol 2006;15: 342-6.

35. Gambichler T, Schlaffke A, Tomi NS, et al. Tacrolimus ointment neither blocks ultraviolet $B$ nor affects expression of thymine dimers and p53 in human skin. J Dermatol Sci 2008;50:115-22.

36. Arellano FM, Wentworth CE, Arana A, et al. Risk of lymphoma following exposure to calcineurin inhibitors and topical steroids in patients with atopic dermatitis. J Invest Dermatol 2007;127:808-16.

37. Margolis DJ, Hoffstad 0, Bilker W. Lack of association between exposure to topical calcineurin inhibitors and skin cancer in adults. Dermatology 2007;214:289-95.

38. Schneeweiss S, Doherty M, Zhu S, et al. Topical treatments with pimecrolimus, tacrolimus and medium- to high-potency corticosteroids, and risk of lymphoma. Dermatology 2009;219:7-21.

39. McNeill AM, Koo JY. "Unknown Risks" of non-steroid topical medications for atopic dermatitis. Int J Dermatol 2007;46:656-8.

40. Rustin MH. The safety of tacrolimus ointment for the treatment of atopic dermatitis: a review. Br J Dermatol 2007;157:86173 .

41. Sørensen HT, Mellemkjaer L, Nielsen GL, et al. Skin cancers and non-hodgkin lymphoma among users of systemic glucocorticoids: a population-based cohort study. J Natl Cancer Inst 2004;96:709-11.

42. Scheinman RI, Cogswell PC, Lofquist AK, Baldwin AS Jr. Role of transcriptional activation of I kappa B alpha in mediation of immunosuppression by glucocorticoids. Science 1995;270:283-6.

43. Auphan N, DiDonato JA, Rosette C, et al. Immunosuppression by glucocorticoids: inhibition of NF-kappa B activity through induction of I kappa B synthesis. Science
1995;27:286-90.

44. Ashworth J, Booker J, Breathnach SM. Effects of topical corticosteroid therapy on Langerhans cell antigen presenting function in human skin. Br J Dermatol 1988; 118:457-69.

45. Fisher LB, Maibach HI. The effect of corticosteroids on human epidermal mitotic activity. Arch Dermatol 1971;103:39-44.

46. Hengge UR, Ruzicka T, Schwartz RA, Cork MJ. Adverse effects of topical glucocorticosteroids. J Am Acad Dermatol 2006;54:115.

47. Lubach D, Bensmann A, Bornemann U. Steroid-induced dermal atrophy. Investigations on discontinuous application. Dermatologica 1989;179:67-72.

48. Morman MR. Possible side effects of topical steroids. Am Fam Physician 1981;23: 171-4.

49. Kligman LH, Crosby MJ. Topical tretinoin enhances corticosteroid-induced inhibition of tumorigenesis in hairless mice previously exposed to solar simulating radiation. Cancer Lett 1996;107:217-22.

50. Lowe NJ, Connor MJ, Breeding J, Chalet M. Inhibition of ultraviolet-B epidermal ornithine decarboxylase induction and skin carcinogenesis in hairless mice by topical indomethacin and triamcinolone acetonide. Cancer Res 1982;42:3941-3.

51. Bissett DL, Chatterjee R, Hannon DP. Photoprotective effect of topical antiinflammatory agents against ultraviolet radiation-induced chronic skin damage in the hairless mouse. Photodermatol Photoimmunol Photomed 1990;7:153-8.

52. Lerche CM, Philipsen PA, Poulsen T, Wulf HC. Topical hydrocortisone, clobetasol propionate, and calcipotriol do not increase photocarcinogenesis induced by simulated solar irradiation in hairless mice. Exp Dermatol 2010 Jan 25.
53. Pashko LL, Rovito RJ, Williams JR, et al. Dehydroepiandrosterone (DHEA) and 3 beta-methylandrost-5-en-17-one: inhibitors of 7,12-dimethylbenz [a] anthracene (DMBA)-initiated and 12-0-tetradecanoylphorbol-13-acetate (TPA)-promoted skin papilloma formation in mice. Carcinogenesis 1984;5:463-6.

54. Menter A, Griffiths CE. Current and future management of psoriasis. Lancet 2007; 370:272-84.

55. Kragballe K. Treatment of psoriasis by the topical application of the novel cholecalciferol analogue calcipotriol (MC 903). Arch Dermatol 1989;125:1647-52.

56. De Haes P, Garmyn M, Verstuyf A, et al. 1,25-Dihydroxyvitamin D3 and analogues protect primary human keratinocytes against UVB-induced DNA damage. J Photochem Photobiol B 2005;78:141-8.

57. Dixon KM, Deo SS, Wong G, et al. Skin cancer prevention: a possible role of 1,25dihydroxyvitamin D3 and its analogs. J Steroid Biochem Mol Biol 2005;97:137-43.

58. Wood AW, Chang RL, Huang MT, et al. 1 alpha, 25-Dihydroxyvitamin D3 inhibits phorbol ester-dependent chemical carcinogenesis in mouse skin. Biochem Biophys Res Commun 1983;116:605-11.

59. Wood AW, Chang RL, Huang MT, et al. Stimulatory effect of 1 alpha, 25-dihydroxyvitamin D3 on the formation of skin tumors in mice treated chronically with 7,12-dimethylbenz [ a ] anthracene. Biochem Biophys Res Commun 1985;130: 924-31.

60. Lerche CM, Philipsen PA, Poulsen T, Wulf HC. Topical hydrocortisone, clobetasol propionate, and calcipotriol do not increase photocarcinogenesis induced by simulated solar irradiation in hairless mice. Exp Dermatol 2010 [Epub ahead of print]. 